\title{
Optimization of Acquisition and Analysis Methods for Clinical Dynamic Susceptibility Contrast MRI Using a Population-Based Digital Reference Object
}

\author{
(D) N.B. Semmineh, (DL.C. Bell, (D)A.M. Stokes, (D).S. Hu, (D).L. Boxerman, and (D)C.C. Quarles
}

\begin{abstract}
BACKGROUND AND PURPOSE: The accuracy of DSC-MR imaging CBV maps in glioblastoma depends on acquisition and analysis protocols. Multisite protocol heterogeneity has challenged standardization initiatives due to the difficulties of in vivo validation. This study sought to compare the accuracy of routinely used protocols using a digital reference object.
\end{abstract}

MATERIALS AND METHODS: The digital reference object consisted of approximately 10,000 simulated voxels recapitulating typical signal heterogeneity encountered in vivo. The influence of acquisition and postprocessing methods on CBV reliability was evaluated across 6912 parameter combinations, including contrast agent dosing schemes, pulse sequence parameters, field strengths, and postprocessing methods. Accuracy and precision were assessed using the concordance correlation coefficient and coefficient of variation.

RESULTS: Across all parameter space, the optimal protocol included full-dose contrast agent preload and bolus, intermediate $\left(60^{\circ}\right)$ flip angle, 30-ms TE, and postprocessing with a leakage-correction algorithm (concordance correlation coefficient $=0.97$, coefficient of variation $=6.6 \%)$. Protocols with no preload or fractional dose preload and bolus using these acquisition parameters were generally less robust. However, a protocol with no preload, full-dose bolus, and low $\left(30^{\circ}\right)$ flip angle performed very well (concordance correlation coefficient $=$ 0.93 , coefficient of variation $=8.7 \%$ at $1.5 \mathrm{~T}$ and concordance correlation coefficient $=0.92$, coefficient of variation $=8.2 \%$ at $3 \mathrm{~T}$ ).

CONCLUSIONS: Schemes with full-dose preload and bolus maximize CBV accuracy and reduce variability, which could enable smaller sample sizes and more reliable detection of CBV changes in clinical trials. When a lower total contrast agent dose is desired, use of a low flip angle, no preload, and full-dose bolus protocol may provide an attractive alternative.

ABBREVIATIONS: ASFNR = American Society of Functional Neuroradiology; API = accuracy and precision index; BSW = Boxerman-Schmainda-Weisskoff; BTIP = brain tumor imaging protocol; $\mathrm{CCC}=$ concordance correlation coefficient; $\mathrm{CV}=$ coefficient of variation; $\mathrm{DRO}=$ digital reference object; FA $=$ flip angle; $\mathrm{GV}=$ $\gamma$-variate fit; rCBV $=$ relative $C B V$

D SC-MR imaging CBV mapping has been used in the brain since the early $1990 \mathrm{~s}^{1,2}$ with multiple neuro-oncology applications, including distinction of tumor from nontumor, glioma grading, biopsy guidance, and assessing response to treatment. ${ }^{3-17}$ However, disagreement persists about the best way to perform DSC-MR imaging and stems primarily from contrast

Received March 8, 2018; accepted after revision June 8.

From the Department of Imaging Research (N.B.S., L.C.B., A.M.S., C.C.Q.), Barrow Neurological Institute, Phoenix, Arizona; Department of Radiology (L.S.H.), Mayo Clinic Arizona, Phoenix, Arizona; and Department of Diagnostic Imaging (J.L.B.), Rhode Island Hospital, Providence, Rhode Island.

This work was supported by the National Institutes of Health/National Cancer Institute (R01 CA213158), the Arizona Biomedical Research Commission (ADHS16162414), and the Barrow Neurological Foundation (R01CA221938, C.C.Q., L.S.H., J.L.B., and U01CA220378, L.S.H.)

Please address correspondence to Chad Quarles, PhD, Barrow Neurological Institute, 350 W Thomas Rd, Phoenix, AZ 85013; e-mail: Chad.Quarles@BarrowNeuro.org; @nbsemmin

- Indicates open access to non-subscribers at www.ajnr.org

http://dx.doi.org/10.3174/ajnr.A5827 agent extravasation through blood-brain barrier deficiencies characteristic of high-grade gliomas. This contrast agent extravasation introduces both pronounced $\mathrm{T} 1$ effects and magnetic susceptibility differences between tumor cells and the extravascular extracellular space, thereby rendering DSC-MR imaging signal a reflection not only of the vascular volume fraction but also cell volume fraction and the vascular permeability. Although sensitivity to these additional physiologic attributes has enabled appropriately acquired DSC-MR imaging to distinguish tumor types, ${ }^{18}$ it has confounded the accuracy of CBV measures, prompting the development of leakage-correction strategies including preload contrast agent administration, pulse sequence acquisition parameters balancing $\mathrm{T} 1$ and $\mathrm{T} 2{ }^{*}$ sensitivities, and postprocessing techniques for correcting the leakage-contaminated signal. ${ }^{19-22}$

The National Cancer Institute (Quantitative Imaging Network; https://imaging.cancer.gov/programs_resources/specialized_ initiatives/qin.htm), the Radiological Society of North America (Quantitative Imaging Biomarkers Alliance; https://www.rsna.org/ 
QIBA/), and the National Brain Tumor Society (Jumpstarting Brain Tumor Drug Development Coalition Imaging Standardization Steering Committee; https://www.itnonline.com/content/newstandardized-brain-tumor-mri-recommendations-announced) have tried to standardize DCE-MR imaging methodology. The American Society of Functional Neuroradiology (ASFNR) published a white paper with a recommended DSC-MR imaging protocol based on practice patterns and literature data. ${ }^{23}$ Such effort is challenged by the relative paucity of data from systematic evaluations of the influence of DSC-MR imaging methodology on CBV accuracy and has relied on anecdotal evidence, including the cor-

Table 1: Summary of investigated parameter space ${ }^{\mathrm{a}}$

\begin{tabular}{ll}
\hline \multicolumn{1}{c}{ Parameter } & \multicolumn{1}{c}{ Values } \\
\hline Preload + bolus (fractional dose) & $(0+1),(1 / 4+3 / 4),(1 / 2+1 / 2)$, \\
& $(1 / 4+1),(1 / 2+1),(1+1)$ \\
Flip angle & $30^{\circ}, 60^{\circ}, 90^{\circ}$ \\
TR $(\mathrm{sec})$ & $1.0,1.5,2.0$ \\
TE $(\mathrm{ms})$ & $20,30,40,50$ \\
Field strength & $1.5 T, 3 \mathrm{~T}$ \\
Upper time limit, integration (sec) & $180,150,120,90$ \\
Postprocessing leakage correction & Uncorrected, BSW, BD, GV, BLS \\
\hline
\end{tabular}

Note:-BD indicates bidirectional; BLS, baseline subtraction.

${ }^{a}$ The dose values are presented as a fraction of a standard contrast agent dose (0.1 $\mathrm{mmol} / \mathrm{kg})$.

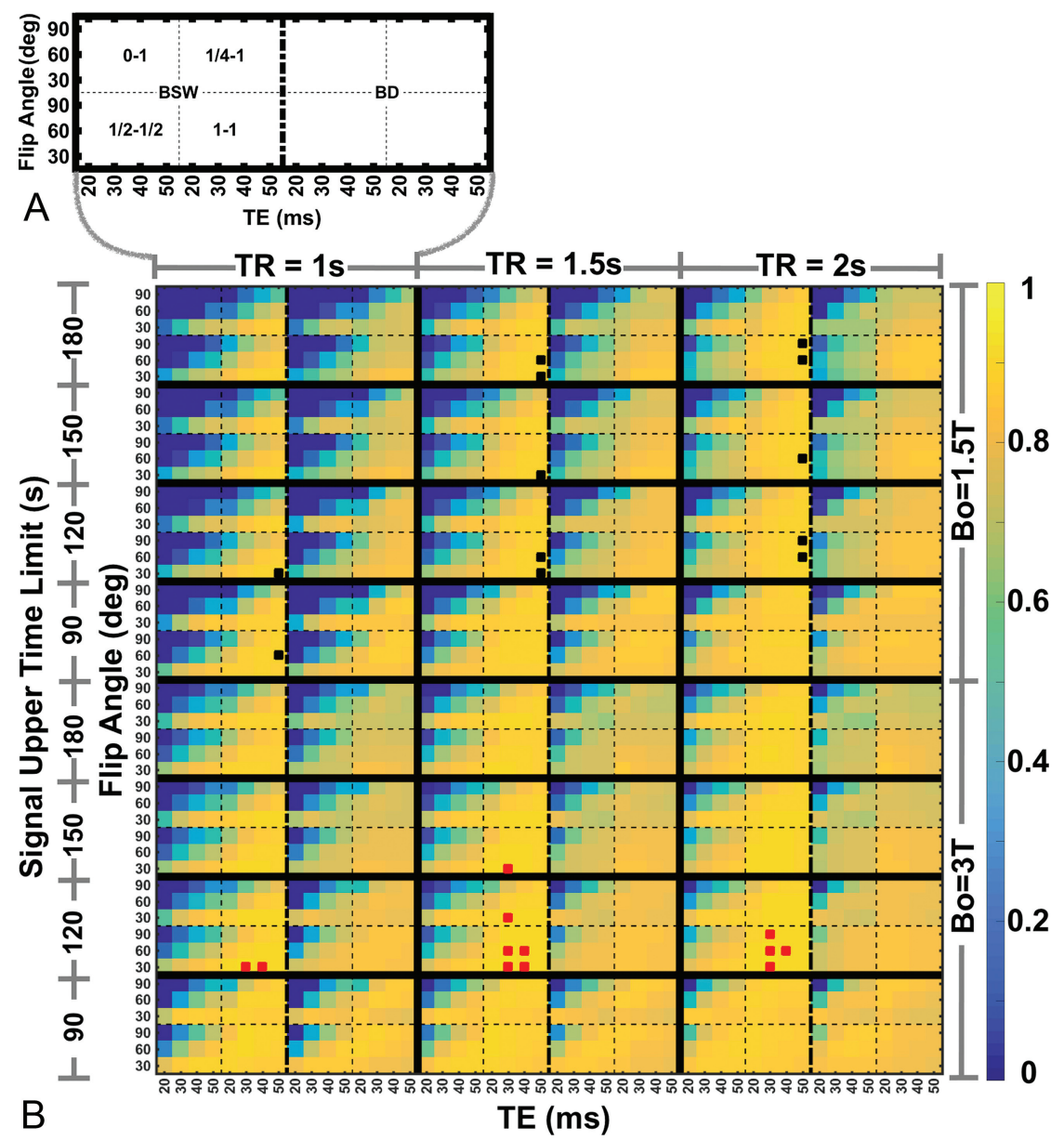

FIG 1. A, Parameter space encompassing 3 flip angles, 4 TEs, 4 contrast agent preload and bolus dose combinations, and 2 postprocessing leakage-correction methods. $B$, Heat map shows CBV accuracy and the precision index for the parameter space shown in $A$ repeated for 3 TRs, $2 B_{0}$ values, and 4 data truncation time points. The top 1\% best-performing parameter combinations are indicated by black $\left(B_{0}=1.5 T\right)$ and red $\left(B_{0}=3 T\right)$ marks. relation of $\mathrm{CBV}$ with glioma grade, ${ }^{24}$ fractional tumor burden in cases of postradiation enhancement, ${ }^{16}$ and patient survival in the setting of conventional and antiangiogenic therapies. ${ }^{12}$ Stereotactic biopsies with coregistered CBV maps have demonstrated general concordance between CBV estimates and histology. While prior in vivo studies evaluated the impact of DSC-MR imaging acquisition parameters and methodologies on CBV data in a small number of patients with high-grade gliomas, ${ }^{25}$ their influence on the underlying CBV accuracy has not been evaluated, likely due to the impracticality of multiple bolus injections in individual patients.

To facilitate the rigorous evaluation of the impact of DSC-MR imaging parameters on CBV accuracy, we recently developed and validated a population-based digital reference object (DRO) that recapitulates for glioblastoma the biophysical basis and inter- and intrasubject heterogeneity of DSC-MR imaging data. ${ }^{26}$ The DRO consists of DSC-MR imaging signals computed across a range of relevant physiologic and acquisition parameters, and clinical relevance was ensured through use of training and validation datasets acquired from multiple patients with glioblastomas. Accordingly, the DRO provides a reliable ground truth for determining the impact of DSC-MR imaging acquisition and postprocessing methods on CBV accuracy. In this study, we used the DRO to evaluate the accuracy and precision of routinely used DSC-MR imaging protocols for glioblastoma, in which the choice of acquisition parameters, including preload and bolus dose, flip angle (FA), TE, and TR, can greatly impact the sensitivity of the acquired signals on $\mathrm{T} 1$ and $\mathrm{T} 2{ }^{*}$ contrast agent leakage effects. Additionally, the accuracy of postprocessing leakage-correction methods ${ }^{24,27}$ may also depend on the degree of $\mathrm{T} 1$ and $\mathrm{T} 2{ }^{*}$ contrast agent leakage effects, further affecting CBV accuracy. Given the interrelated nature of acquisition and postprocessing methods, we used the DRO to evaluate CBV accuracy across a range of commonly used DSC-MR imaging methods, identifying combinations of acquisition parameters yielding particularly high CBV accuracy and precision and potentially guiding future DSC-MR imaging consensus initiatives.

\section{MATERIALS AND METHODS}

We evaluated CBV fidelity across a range of relevant DSC-MR imaging parameters using a validated DRO that computes MR imaging signal intensities for heterogeneous 3D tissue structures, accounting for the relevant underlying DSC-MR imaging contrast mechanisms. ${ }^{26}$ Parameter inputs were ex- 
Table 2: Comparison of the accuracy and precision index, coefficient of variation, and concordance correlation coefficient for all dosing schemes using intermediate and low flip angle protocols at 1.5T and 3T

\begin{tabular}{|c|c|c|c|c|c|c|c|c|c|c|c|c|}
\hline \multirow{3}{*}{$\begin{array}{l}\text { Dosing Scheme } \\
\text { (Preload + Bolus) }\end{array}$} & \multicolumn{6}{|c|}{ Intermediate Flip Angle ${ }^{a}$} & \multicolumn{6}{|c|}{ Low Flip Angle ${ }^{b}$} \\
\hline & \multicolumn{3}{|c|}{$1.5 \mathrm{~T}$} & \multicolumn{3}{|c|}{$3 T$} & \multicolumn{3}{|c|}{$1.5 \mathrm{~T}$} & \multicolumn{3}{|c|}{ 3T } \\
\hline & $\mathrm{CCC}$ & $\mathrm{CV} \%$ & API & $\mathrm{CCC}$ & $\mathrm{CV} \%$ & API & $\mathrm{CCC}$ & $\mathrm{CV} \%$ & API & $\mathrm{CCC}$ & $\mathrm{CV} \%$ & API \\
\hline$(0+1)$ & 0.30 & 21.7 & 0.08 & 0.55 & 12.4 & 0.43 & 0.93 & 8.7 & 0.84 & 0.92 & 8.2 & 0.83 \\
\hline$(1 / 4+3 / 4)$ & 0.51 & 13.3 & 0.38 & 0.74 & 8.8 & 0.65 & 0.94 & 8.9 & 0.85 & 0.94 & 7.8 & 0.86 \\
\hline$(1 / 2+1 / 2)$ & 0.61 & 12.7 & 0.48 & 0.76 & 9.2 & 0.67 & 0.91 & 10.6 & 0.80 & 0.90 & 8.7 & 0.82 \\
\hline$(1 / 4+1)$ & 0.86 & 7.9 & 0.78 & 0.93 & 6.8 & 0.86 & 0.96 & 7.7 & 0.89 & 0.97 & 7.0 & 0.90 \\
\hline$(1 / 2+1)$ & 0.90 & 7.7 & 0.82 & 0.96 & 6.8 & 0.89 & 0.96 & 7.5 & 0.89 & 0.97 & 6.8 & 0.91 \\
\hline$(1+1)$ & 0.94 & 7.4 & 0.86 & 0.97 & 6.6 & 0.91 & 0.96 & 7.4 & 0.89 & 0.98 & 6.8 & 0.91 \\
\hline
\end{tabular}

${ }^{\mathrm{a}} \mathrm{FA}=60^{\circ}, \mathrm{TE}=30 \mathrm{~ms}, \mathrm{TR}=1.5 \mathrm{sec}$.

${ }^{\mathrm{b}} \mathrm{FA}=30^{\circ}, \mathrm{TE}=50 \mathrm{~ms}, \mathrm{TR}=1.5 \mathrm{sec}, \mathrm{B}_{0}=1.5 \mathrm{~T} ; \mathrm{FA}=30^{\circ}, \mathrm{TE}=30 \mathrm{~ms}, \mathrm{TR}=1.5 \mathrm{sec}, \mathrm{B}_{0}=3 \mathrm{~T}$.

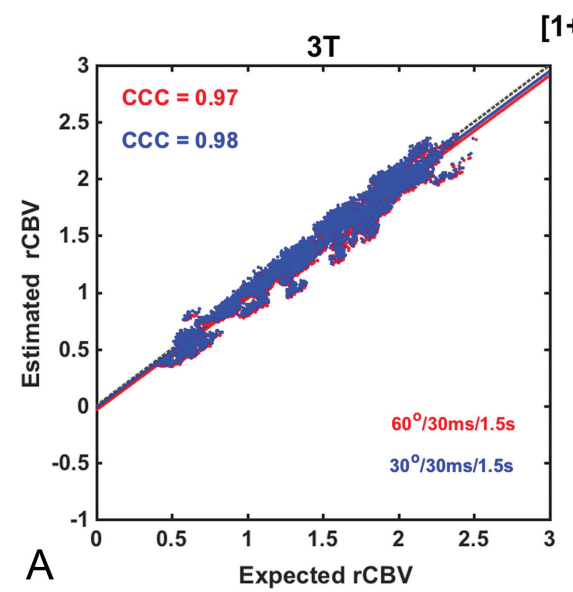

$[1+1]$
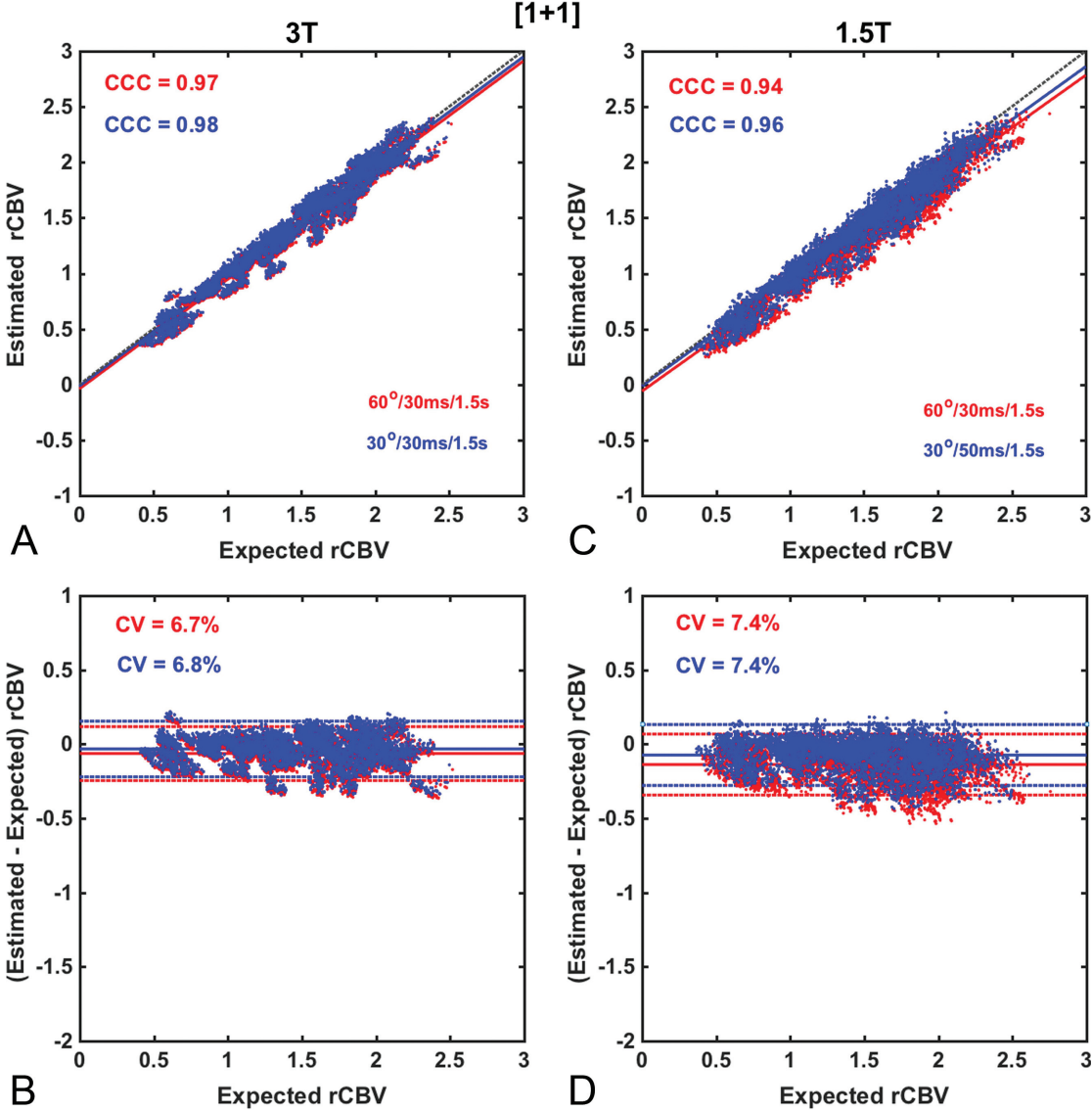

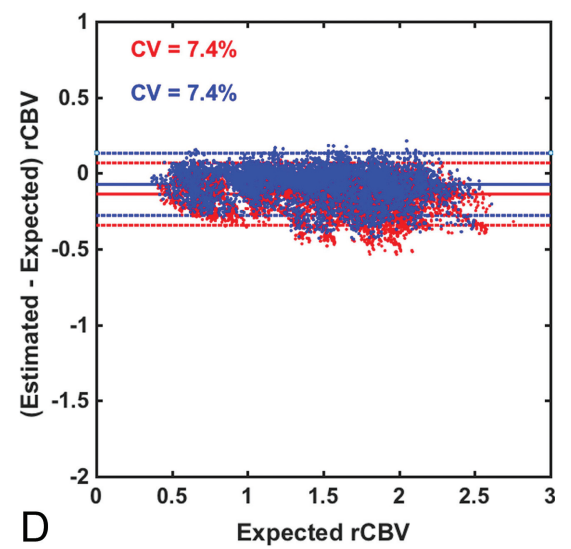

FIG 2. Scatter- and Bland-Altman plots comparing CBV estimates for the $(1+1)$ dosing scheme at 3T ( $A$ and $B$ ) and at 1.5T ( $C$ and $D$ ) using intermediate (red) and low (blue) flip angle protocols. At 3T, both protocols yield equivalent accuracy and precision ( $\mathrm{API}=0.91$ ), whereas at $1.5 \mathrm{~T}$, the low flip angle protocol provides slightly higher accuracy and precision (API $=0.89$ ) compared with the use of an intermediate flip angle (API $=0.86$ ). Leakage correction was applied using BSW.

tracted from voxelwise training data from 23 patients with glioblastomas ( $>40,000$ voxels). To ensure that the simulated signals accurately represented the magnitude and distribution of contrast agent-induced $\mathrm{T} 1$ and $\mathrm{T} 2^{*}$ changes within typical glioblastomas, we chose model parameters so that the distribution of percentage signal recovery and the mean SD of signal intensities across the DRO matched those in the patient training dataset. The training process yielded approximately 10,000 DRO voxels that agreed with the in vivo data. The DRO was validated against a separate set of patients with glioblastomas imaged with pulse sequences and contrast agent dosing schemes differing from those in the training dataset.
To investigate the influence of acquisition and postprocessing methods on the reliability of CBV measurements, we compared tumor CBV simulated with and without contrast agent leakage effects for the most commonly used DSC-MR imaging methods, including the following: 6 preload and bolus injection combinations, 3 flip angles, 3 TRs, 2 $\mathrm{B}_{0}$ values, 4 TEs, and 4 upper time limits for postprocessing integration (with a constant prebolus time). Furthermore, we compared the uncorrected $\mathrm{CBV}$ estimates with 4 postprocessing leakage-correction methods: Boxerman-Schmainda-Weisskoff (BSW), ${ }^{24}$ bidirectional, ${ }^{27} \gamma$-variate fit, and postbolus baseline subtraction. ${ }^{25}$ Note that while baseline subtraction correction is typically applied to low flip angle data that predominantly exhibit $\mathrm{T} 2{ }^{*}$ leakage effects, we implemented a version that corrects both $\mathrm{T} 1$ and $\mathrm{T} 2{ }^{*}$ effects. Because baseline subtraction correction arbitrarily forces all signals to return to baseline, it was applied to both normal and tumor signals in the DRO. The investigated parameter space is summarized in Table 1. Across all parameter space, the concordance correlation coefficient (CCC) and the coefficient of variation $(\mathrm{CV})$ between the simulated tumor $\mathrm{CBV}$ and the ground truth (the CBV values not confounded by leakage effects) were computed as a marker of accuracy and precision, respectively. To aid in the identification of the optimal protocol, we computed an accuracy and precision index (API) that combines the CCC and CV: $\mathrm{AP}=\mathrm{CCC}-|\mathrm{CV}| / 100$. Accordingly, API values of $1(\mathrm{CCC}=1$, $\mathrm{CV} / 100=0)$ reflect the ideal agreement. Protocols compliant with a recently published consensus brain tumor imaging protocol (BTIP) ${ }^{28}$ were highlighted because these can be most easily incorporated into clinical trial imaging protocols. Note that dosing schemes that are labeled "BTIP compliant" are those that align with the recommended dosing requirements and not necessarily the timing between the contrast agent injection and the postcontrast T1-weighted scan. 

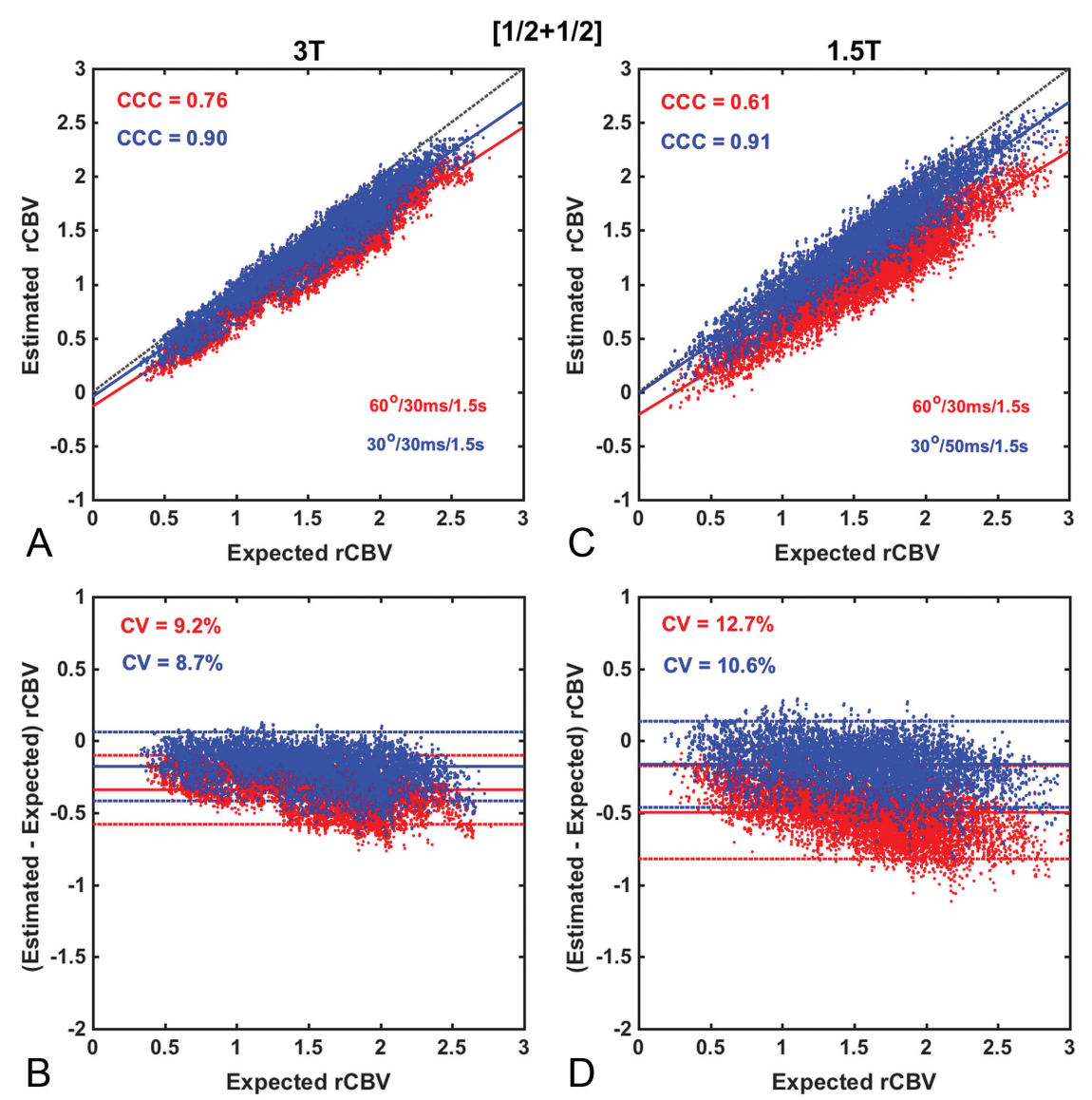

FIG 3. Scatter- and Bland-Altman plots comparing CBV estimates for the $(1 / 2+1 / 2)$ dosing scheme at $3 \mathrm{~T}(A$ and $B)$ and at 1.5T ( $C$ and $D)$ using the intermediate (red) and low flip angle (blue) protocols. At both $3 \mathrm{~T}$ and 1.5T, CBV values measured using an intermediate flip angle were consistently underestimated, yielding lower accuracy and precision (API $=0.67$ and 0.48 ), respectively. The use of the low flip angle protocol improved both CBV accuracy and precision at 3T $(\mathrm{API}=0.82)$ and $1.5 \mathrm{~T}(\mathrm{API}=0.80)$. Leakage correction was applied using BSW.

\section{RESULTS}

Figure 1 shows the API heat map across the parameter space in Table 1 . The heat map in Fig 1 is segmented horizontally by $\mathrm{B}_{0}$ (top half: $\mathrm{B}_{0}=1.5 \mathrm{~T}$; bottom half: $\mathrm{B}_{0}=3 \mathrm{~T}$ ). Within each $\mathrm{B}_{0}$ segment, the heat map is further segmented vertically by the acquisition time limit (4 segments) and horizontally by TR (3 segments). For each acquisition time limit and TR combination, the heat map is further segmented, as shown in Fig $1 A$, horizontally by the leakage-correction method (2 segments for BSW and bidirectional; $\gamma$-variate fit, baseline subtraction, and uncorrected are excluded). Finally, each leakage-correction segment is split into 4 subplots $(2 \times 2)$ by contrast agent dosing [clockwise from top left: $(0+1),(1 / 4+1),(1+1),(1 / 2+1 / 2)]$, where the first and second numeric values $[\mathrm{eg},(1+1)]$ represent the preload and bolusinjection dose as a fraction of a total standard dose $(0.1 \mathrm{mmol} / \mathrm{kg})$. Each of these subplots shows the variation horizontally by TE (4 squares) and vertically by flip angle (3 squares). This singular figure demonstrates the API for 2304 possible parameter combinations (of the 8640 tested). The uncorrected $\gamma$-variate fit and baseline subtraction results are not included in Fig $1 B$ (or in subsequent figures or tables) because their associated CCC, CV, and API values were substantially lower than those for BSW and bidirectional. For simplicity, the $(1 / 4+3 / 4)$ and $(1 / 2+1)$ contrast agent dosing schemes were not incorporated in the heat map, but their results are summarized in Table 2.

Several results can be observed from the heat map. First, at both field strengths, $(1+1)$ contrast agent dosing provides the highest API and the least sensitivity (more consistent API values) to variations in TR, TE, and flip angle, compared with other dosing schemes. Across all parameter combinations, the top $1 \%$ of API values $(>0.89)$ are derived from protocols that rely on $(1+1)$ contrast agent dosing at both field strengths, as indicated by the black (1.5T) and red (3T) boxes. Although the top $1 \%$ of parameter combinations incorporate BSW leakage correction, both BSW and bidirectional leakage correction methods yielded similar APIs. Note that the top $1 \%$ is an arbitrary threshold, and the difference in utility of CBV maps derived from methods yielding slightly lower APIs (eg, top $1 \%$ versus the top $3 \%-5 \%$ ) may not be clinically meaningful. Second, the API varies more substantially for acquisitions using $(0+1)$ and $(1 / 2+1 / 2)$ contrast agent dosing schemes, indicating greater sensitivity to contrast agent leakage effects and CNR losses related to low contrast agent dose. Third, for data acquired without or with small contrast agent preloads, a low flip angle $\left(30^{\circ}\right)$ maximizes the API. Fourth, the number of data points incorporated after the first pass into the CBV acquisition and analysis has minimal impact on API values, with a slight preference for shorter data lengths using the BSW correction.

Figures 2-5 compare the accuracy and precision of CBV measures using scatterplots (top) and Bland-Altman plots (bottom) for commonly used contrast agent dosing protocols [Fig 2: $(1+$ 1), Fig 3: $(1 / 2+1 / 2)$, Fig 4: $(1 / 4+1)$, Fig $5:(0+1)]$, field strengths $\left(1.5 \mathrm{~T}\right.$ and $3 \mathrm{~T}$ ), and flip angles (low $30^{\circ}$ versus intermediate $60^{\circ}$ ). The BSW leakage-correction method was applied to all data. For each flip angle, the TE was selected to maximize the API, yielding 4 parameter combinations: $1.5 \mathrm{~T}, \mathrm{FA} / \mathrm{TE}=30^{\circ} / 50 \mathrm{~ms}$ and $\mathrm{FA} /$ $\mathrm{TE}=60^{\circ} / 30 \mathrm{~ms} ; 3 \mathrm{~T}, \mathrm{FA} / \mathrm{TE}=30^{\circ} / 30 \mathrm{~ms}$ and $\mathrm{FA} / \mathrm{TE}=60^{\circ} / 30 \mathrm{~ms}$. The first goal of this analysis was to evaluate CBV accuracy when data are acquired using the ASFNR parameter recommendations (represented by the intermediate flip angle protocol) and variable dosing protocols and field strengths. The second was to compare this performance with that derived from the low flip angle case, which provided the overall highest CBV accuracy and precision.

Across the entire range of parameters, the highest CBV accuracy and precision (API $=0.91$ ) was found for DSC-MR imaging data collected at 3T with a $(1+1)$ dosing scheme and either an intermediate or low flip angle (Fig 2). At 1.5T, the CCC and CV 

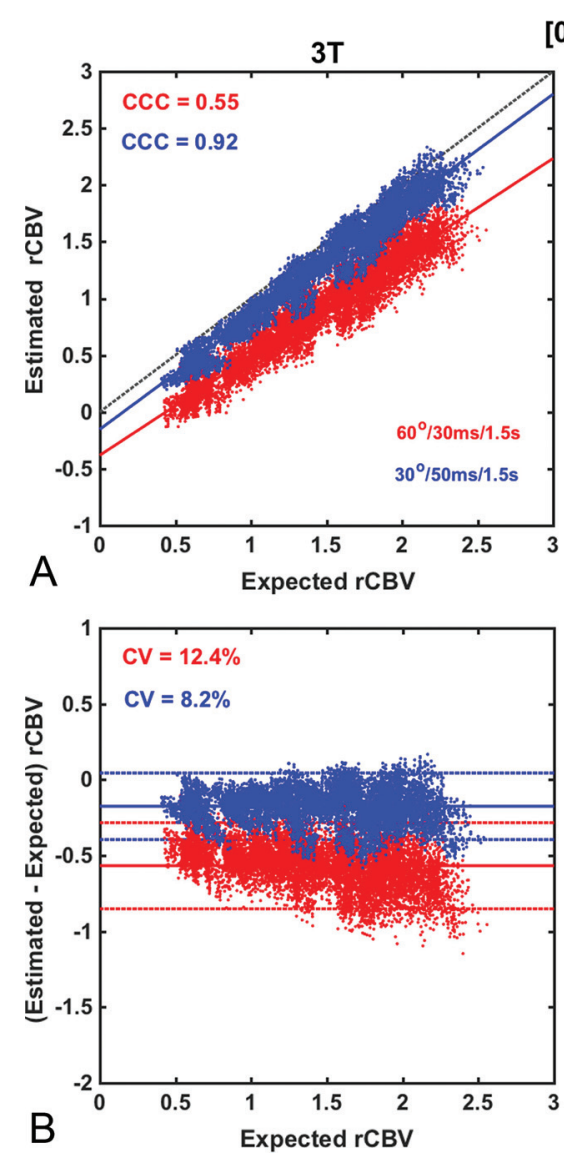

$1.5 \mathrm{~T}$
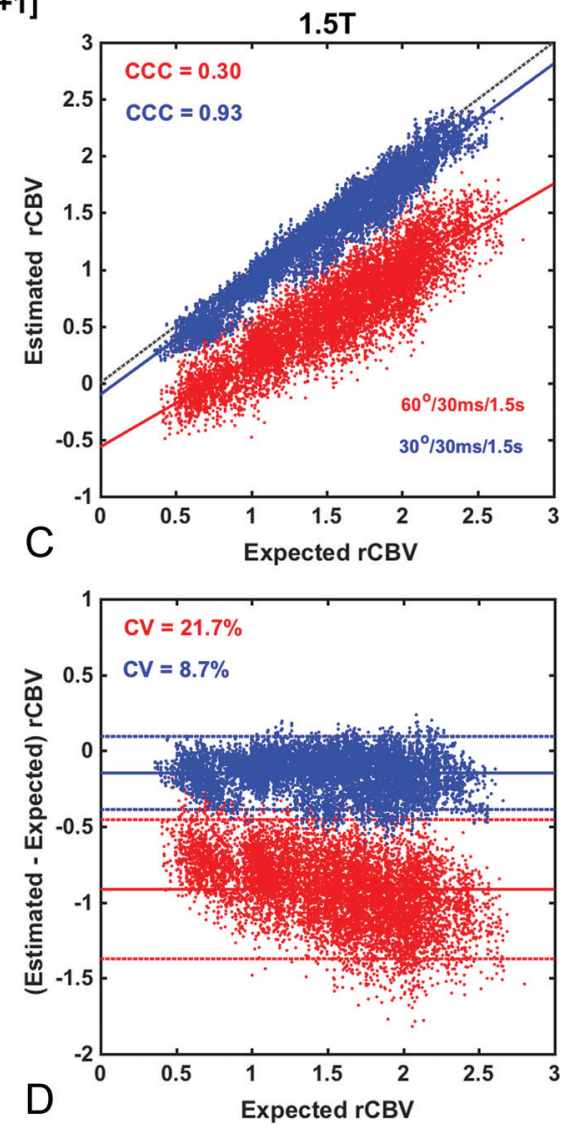

FIG 4. Scatter- and Bland-Altman plots comparing CBV estimates for the $(0+1)$ dosing scheme at 3T $(A$ and $B$ ) and at 1.5T ( $C$ and $D$ ), using the intermediate (red) and low (blue) flip angle protocols. At both 3T and 1.5T, CBV measured using an intermediate flip angle yielded poor accuracy and precision (API $=0.43$ and 0.08 ), respectively. Whereas the use of a low flip angle substantially improved $\mathrm{CBV}$ reliability at both $3 \mathrm{~T}(\mathrm{API}=0.83)$ and $1.5 \mathrm{~T}(\mathrm{API}=0.84)$, respectively. Leakage correction was applied using BSW.

values were only slightly lower than those at 3T, yielding similar API values ( 0.86 and 0.89 for intermediate and low flip angles, respectively).

For the $(1 / 2+1 / 2)$ dosing scheme, illustrated in Fig 3 , CBV measured using the intermediate flip angle protocol was consistently underestimated due to residual (uncorrected) T1 leakage effects, yielding moderately lower accuracy and precision (API $=0.67)$ at $3 \mathrm{~T}$ and poor performance $(\mathrm{API}=0.48)$ at $1.5 \mathrm{~T}$. The use of a low flip angle improved the API at both $3 \mathrm{~T}(\mathrm{API}=0.82)$ and $1.5 \mathrm{~T}(\mathrm{API}=$ $0.80)$ due to reduced T1 sensitivity. However, the API for this dosing scheme was consistently lower than the $(1+1)$ dosing scheme.

As illustrated in Fig 4, the use of an intermediate flip angle at $3 \mathrm{~T}$ without a preload $(0+1)$ dosing scheme substantially reduced both accuracy and precision $(\mathrm{API}=0.43)$. This poor performance was exacerbated at $1.5 \mathrm{~T}(\mathrm{API}=0.08)$. These results are consistent with previous studies demonstrating the necessity of preload when using moderate and high flip angles. ${ }^{25,29,30}$ By comparison, the use of the low flip angle protocol at $3 \mathrm{~T}$ without preload introduces a relatively minor penalty $(\mathrm{API}=0.83)$ compared with the $(1+1)$ dosing scheme $(\mathrm{API}=0.91)$. At $1.5 \mathrm{~T}, \mathrm{CBV}$ measured using the low flip angle protocol provided a substantial improvement compared with the intermediate flip angle in both accuracy and precision (API $=0.84)$.

Figure 5 shows CBV accuracy and precision for DSC-MR imaging acquired with $(1 / 4+1)$ contrast agent dosing. At $3 \mathrm{~T}$, both the intermediate and low flip angle protocols provide excellent accuracy (API values of 0.86 and 0.90 , respectively), comparable with that for the $(1+1)$ dosing scheme at $3 \mathrm{~T}$ $(\mathrm{API}=0.91)$. At $1.5 \mathrm{~T}$, the intermediate flip angle protocol yielded intermediate accuracy and precision (API $=0.78$ ), whereas the low flip angle protocol yielded an API value (0.89) comparable with that for the $(1+1)$ dosing scheme at $3 \mathrm{~T}$.

Table 2 compares CCC, CV, and API for the intermediate and low flip angle protocols at both field strengths and all dosing schemes. Across all dosing schemes and field strengths, the low FA provided equivalent or better API values compared with CBV data acquired with an intermediate FA. For BTIP-compliant dosing schemes $(0+1),(1 / 4+3 / 4)$, $(1 / 2+1 / 2)$, and $(1+1)$, the $(1+1)$ dosing yielded the highest accuracy and precision for both intermediate and low FA acquisitions and at both field strengths. With an intermediate FA, single totaldose protocols $(0+1),(1 / 4+3 / 4)$, and $(1 / 2+1 / 2)$ at $1.5 \mathrm{~T}$ and the no preload protocol $(0+1)$ at $3 \mathrm{~T}$ have poor performance, with moderately good performance for split-dose protocols $(1 / 4+3 / 4)$ and $(1 / 2+1 / 2)$ at $3 \mathrm{~T}$. For non-BTIP-compliant dosing schemes $(1 / 4+1)$ and $(1 / 2+1)$, low and intermediate FA acquisitions yielded excellent results at $3 \mathrm{~T}$, and very good results at $1.5 \mathrm{~T}$, comparable with the optimal $(1+$ 1) dosing protocol.

\section{DISCUSSION}

The computational analysis presented herein confirms that it is possible to acquire highly accurate and precise relative CBV (rCBV) measurements in glioblastoma with proper selection of DSC-MR imaging methodology. To date, recommendations on optimal DSC-MR imaging acquisition protocols, such as the ASFNR white paper, ${ }^{23}$ have relied on susceptibility contrast theory, clinical experience, and limited studies involving correlation with stereotactic biopsies, ${ }^{16,31}$ but this study establishes the limits of accuracy and precision using a computational approach across a wide range of parameters and, accordingly, may be used to inform the aforementioned standardization initiatives.

While the DRO used in this study can quantitatively assess rCBV accuracy for any combination of acquisition and postprocessing parameters, the overwhelming number of permutations makes identifying a single optimal approach challenging. For 


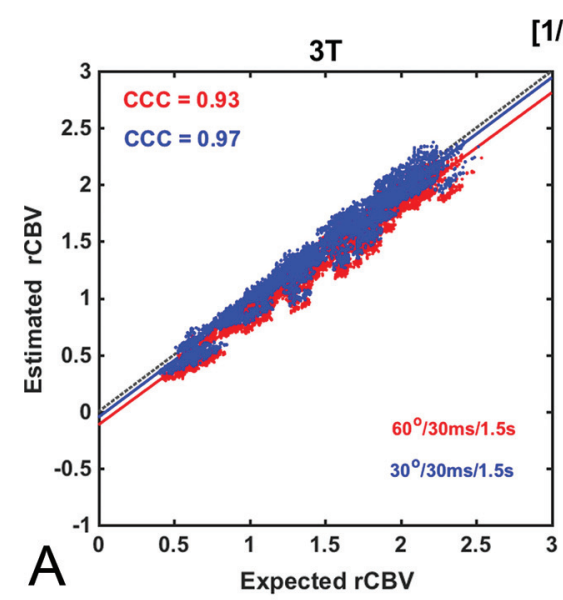

$[1 / 4+1]$

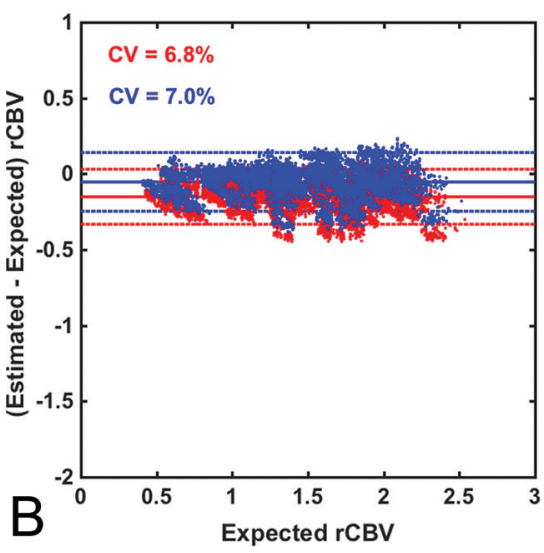

$1.5 T$
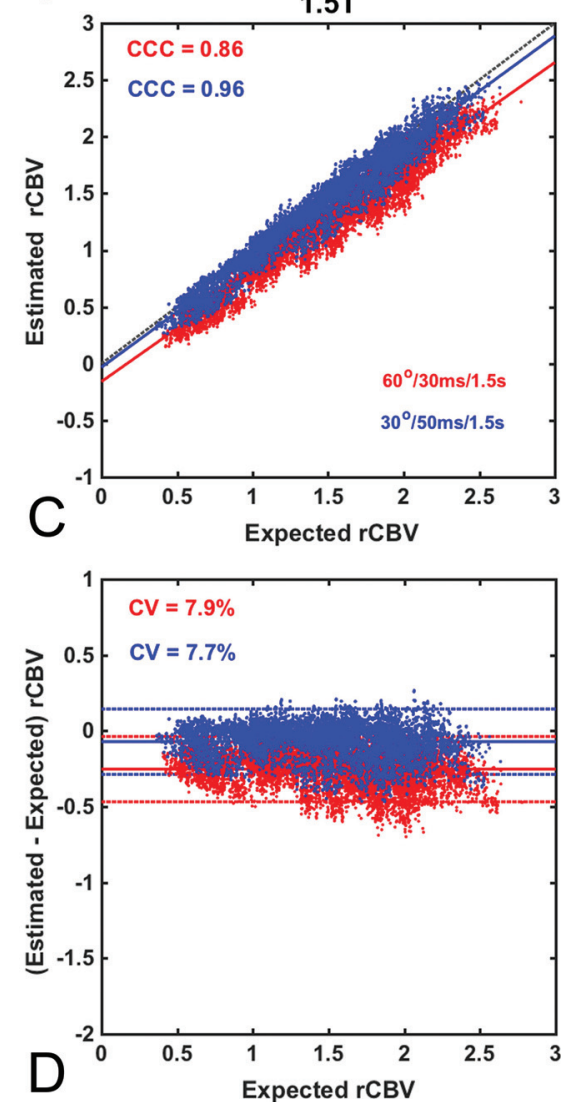

FIG 5. Scatter- and Bland-Altman plots comparing CBV estimates for the $(1 / 4+1)$ dosing scheme at 3T $(A$ and $B$ ) and 1.5T ( $C$ and $D)$ using the intermediate (red) and low (blue) flip angle protocols. At $3 \mathrm{~T}$, both the intermediate and low flip angle protocols provide excellent accuracy (API $=0.86$ and 0.90 , respectively), comparable with that for $(1+1)$ dosing at $3 \mathrm{~T}(\mathrm{API}=0.91)$. Similar accuracy and precision levels were achieved at 1.5T using the low flip angle protocol (API $=0.89$ ), but not the intermediate flip angle (API $=0.78$ ). Leakage correction was applied using BSW.

example, the heat maps illustrate that there are many unique parameter combinations that yield reasonably accurate rCBV estimates. However, for many of these combinations, minor parameter modification (eg, changing flip angles or TEs) yields substantially decreased accuracy and/or precision. Such isolated "local maximums" reflect specific parameter subsets that may suffice for singular distributions of $\mathrm{T} 1$ and $\mathrm{T} 2{ }^{*}$ leakage effects but that are not broadly effective when those distributions are altered, such as greater sensitivity to T1 effects due to higher flip angles. While the DRO captures the full range of $\mathrm{T} 1$ and $\mathrm{T}^{*}$ leakage effects that would be encountered across patients, the distribution of these effects within a single patient could be unique. Accordingly, our strategy for identifying the most robust DSC-MR imaging protocol included accuracy, precision, and consistency across localized parameter space (eg, a consistent API as the TE is varied), ensuring the identification of optimal methods both across and within patients.

Across all parameter space, the combination of preload scheme and acquisition parameters yielding the most robust rCBV estimates included full-dose preload and bolus $(1+1)$, with either the recommended ASFNR (intermediate flip angle) or low flip angle pulse sequence parameters. This finding is consistent with a prior in vivo study that demonstrated that the $(1+1)$ dosing scheme provided the most robust distinction between tumor and normal tissue $\mathrm{rCBV}^{25}$ Most important, this dosing scheme maintains BTIP compliance because the postcontrast T1weighted images can be acquired between preload and bolus contrast agent injections. The $(1+1)$ dosing scheme requires double the standard dose of contrast agent $(0.2 \mathrm{mmol} / \mathrm{kg})$, and given the growing concern for long-term gadolinium deposition within the brain following repeat use of gadolinium-based contrast agents, we also investigated the accuracy of lower-dose schemes. Lowerdose, BTIP-compliant dosing schemes using the intermediate flip angle protocol introduced bias and lower precision compared with the $(1+1)$ scheme. Lower preload doses yielded more error because they are not as effective at reducing T1 leakage effects before the primary bolus injection.

For dosing schemes using less than a full dose for the primary bolus, the relative magnitude of T1 leakage effects with respect to the expected $\mathrm{T} 2{ }^{*}$ changes are larger than would be observed with the $(1+1)$ scheme and appear to partially confound leakage-correction algorithms. A lower primary bolus dose scheme $[\mathrm{eg},(1 / 2+1 / 2)]$ also lowers the contrast-to-noise ratio and decreases precision. This reduction in precision could yield greater within- and across-subject rCBV variability, confounding the assessment of treatment response, determination of thresholds for clinical use (eg, differentiating between tumor recurrence and radiation effects), and the sample size needed for clinical trials. These errors are magnified at $1.5 \mathrm{~T}$ compared with 3T, which has further implications for clinical trial design. Although lower-dose protocols have reduced accuracy and precision, prior single-site studies have demonstrated their clinical utility for differentiating tumor recurrence and posttreatment radiation effects ${ }^{29}$ and detecting rCBV changes following bevacizumab therapy. ${ }^{32}$

Another major finding of this work is that DSC-MR imaging data acquired with a low flip angle provide comparable or improved CBV accuracy across all dosing schemes and field strengths. As highlighted in Table 2, the acquisition protocol using a single total-dose contrast agent administration, $[(0+1)$, $(1 / 4+3 / 4),(1 / 2+1 / 2)]$, which yielded only a slighted reduced API, was a low flip angle scheme $\left(\mathrm{FA}=30^{\circ}, \mathrm{TR}=1.5 \mathrm{sec} ; \mathrm{TE}=50 \mathrm{~ms}\right.$ at $1.5 \mathrm{~T}$ and $\mathrm{TE}=30 \mathrm{~ms}$ at $3 \mathrm{~T}$ ). The corresponding $\mathrm{CCC}$ values exceeded 0.9 at both $1.5 \mathrm{~T}$ and $3 \mathrm{~T}$ but exhibited slightly higher $\mathrm{CV}$ values due to a lower contrast-to-noise ratio compared with the $(1+$ 1) double-dose protocol. The similarity of the API values across 
these single total-dose protocols, when combined with a low flip angle, indicates that the preload does not improve CBV fidelity and may not be needed in clinical practice. The use of a $(0+1)$ dosing scheme with a low FA has the benefit of maintaining BTIP compliance and requiring only a single bolus injection, thereby simplifying perfusion scan protocols and increasing their consistency across patients and clinical trial sites. Given the potential ramifications of this finding on DSC-MR imaging recommendations, it would be highly relevant to compare, in the same patients, the agreement and CBV map quality from data acquired with this low flip angle alternative and the ASFNR recommendations (using the double-dose protocol). Given that DSC-MR imaging data acquired with $\mathrm{TE}=50 \mathrm{~ms}$ (the optimal TE at 1.5T) could be more sensitive to EPI-related artifacts and exhibit lowered contrast to noise, in vivo studies should be performed to confirm CBV map quality and interpretability. Paulson and Schmainda ${ }^{25}$ found that the use of a low flip angle and a 50-ms TE reduced the difference between tumor and normal tissue rCBV.

Similar to clinical results, ${ }^{25}$ leakage-correction models based on contrast agent kinetics, such as the BSW or bidirectional methods, provide the highest rCBV accuracy compared with no leakage correction or arbitrary curve fitting, like $\gamma$-variate. While the latter provides curves that, in appearance, resemble traditional firstpass data, they cannot reliably correct the signal because of the underlying complexity of simultaneous and competing T1 and $\mathrm{T}^{*}$ leakage effects. The highest accuracy and precision were found when DSC-MR imaging data were corrected using the BSW approach. The bidirectional correction was similarly accurate but with lower precision. In future work, we will expand these studies to investigate model-independent and MTT-insensitive leakagecorrection methods ${ }^{19,33,34}$ and methods that rely on biophysical models applied to multiecho data. ${ }^{35,36}$

\section{CONCLUSIONS}

By leveraging a validated DRO, we characterized the influence of DSC-MR imaging methodology on CBV accuracy and precision and identified optimal protocols for clinical use. Our results support, to a large extent, the ASFNR recommendations on DSC-MR imaging best practices, ${ }^{23}$ particularly at $3 \mathrm{~T}$ and when paired with the $(1+1)$ dosing protocol, which should encourage its broader acceptance and standardization. Taken together, the results indicate that for clinical trials, a $(1+1)$ dosing scheme provides highly accurate and consistent CBV estimates, enabling smaller sample sizes for detecting treatment-related changes in CBV. For clinical surveillance scans, in which a lower total gadolinium dose is desirable, the low flip angle option without preload or the $(1 / 4+1)$ dosing scheme is a viable alternative, providing reasonably robust $\mathrm{CBV}$ measures. An added advantage of these schemes is that they perform well at both $1.5 \mathrm{~T}$ and $3 \mathrm{~T}$. Such considerations may inform standardization initiatives for the acquisition and analysis of DSC-MR imaging data in clinical trials and routine patient care.

Disclosures: Natenael B. Semmineh—RELATED: Grant: National Institutes of Health, Arizona Biomedical Research Commission, and Barrow Neurological Foundation, Comments: This work was supported by National Institutes of Health/National Cancer Institute (R01 CA213158), the Arizona Biomedical Research Commission (ADHS16162414), and the Barrow Neurological Foundation.* Christopher C. QuarlesRELATED: Grant: National Institutes of Health. ${ }^{\star}$ Leland S. Hu—RELATED: Grant: National Institutes of Health/National Cancer Institute. * Money paid to the institution.

\section{REFERENCES}

1. Belliveau JW, Kennedy DN Jr, McKinstry RC, et al. Functional mapping of the human visual cortex by magnetic resonance imaging. Science 1991;254:716-19 Medline

2. Aronen HJ, Cohen MS, Belliveau JW, et al. Ultrafast imaging of brain tumors. Top Magn Reson Imaging 1993;5:14-24 Medline

3. Prah MA, Al-Gizawiy MM, Mueller WM, et al. Spatial discrimination of glioblastoma and treatment effect with histologically-validated perfusion and diffusion magnetic resonance imaging metrics. J Neurooncol 2018;136:13-21 CrossRef Medline

4. Liu TT, Achrol AS, Mitchell LA, et al. Magnetic resonance perfusion image features uncover an angiogenic subgroup of glioblastoma patients with poor survival and better response to antiangiogenic treatment. Neuro Oncol 2017;19:997-1007 CrossRef Medline

5. Law M, Yang S, Wang H, et al. Glioma grading: sensitivity, specificity, and predictive values of perfusion MR imaging and proton MR spectroscopic imaging compared with conventional MR imaging. AJNR Am J Neuroradiol 2003;24;1989-98 Medline

6. Sugahara T, Korogi Y, Shigematsu Y, et al. Value of dynamic susceptibility contrast magnetic resonance imaging in the evaluation of intracranial tumors. Top Magn Reson Imaging 1999;10:114-124 Medline

7. Lev MH, Rosen BR. Clinical applications of intracranial perfusion MR imaging. Neuroimaging Clin N Am 1999;9:309-31 Medline

8. Aronen HJ, Gazit IE, Louis DN, et al. Cerebral blood volume maps of gliomas: comparison with tumor grade and histologic findings. $\mathrm{Ra}$ diology 1994;191:41-51 Medline

9. Nasseri M, Gahramanov S, Netto JP, et al. Evaluation of pseudoprogression in patients with glioblastoma multiforme using dynamic magnetic resonance imaging with ferumoxytol calls RANO criteria into question. Neuro Oncol 2014;16:1146-54 CrossRef Medline

10. Shiroishi MS, Boxerman JL, Pope WB. Physiologic MRI for assessment of response to therapy and prognosis in glioblastoma. Neuro Oncol 2016;18:467-78 CrossRef Medline

11. Boxerman JL, Ellingson BM. Response assessment and magnetic resonance imaging issues for clinical trials involving high-grade gliomas. Top Magn Reson Imaging 2015;24:127-36 CrossRef Medline

12. Schmainda KM, Zhang Z, Prah M, et al. Dynamic susceptibility contrast MRI measures of relative cerebral blood volume as a prognostic marker for overall survival in recurrent glioblastoma: results from the ACRIN 6677/RTOG 0625 multicenter trial. Neuro Oncol 2015;17:1148-56 CrossRef Medline

13. Harris RJ, Cloughesy TF, Hardy AJ, et al. MRI perfusion measurements calculated using advanced deconvolution techniques predict survival in recurrent glioblastoma treated with bevacizumab. J Neurooncol 2015;122:497-505 CrossRef Medline

14. Boxerman JL, Ellingson BM, Jeyapalan S, et al. Longitudinal DSCMRI for distinguishing tumor recurrence from pseudoprogression in patients with a high-grade glioma. Am J Clin Oncol 2017;40: 228-34 CrossRef Medline

15. Raghavan D, Boxerman J, Rogg J, et al. Glioblastoma multiforme: utilization of advanced MRI techniques for preoperative planning. Med Health R 2012;95:42-43 Medline

16. Hu LS, Heiserman JE, Dueck AC, et al. Reevaluating the imaging definition of tumor progression: perfusion MRI quantifies recurrent glioblastoma tumor fraction, pseudoprogression, and radiation necrosis to predict survival. Neuro Oncol 2012;14:919-30 CrossRef Medline

17. Schmainda KM, Rand SD, Joseph AM, et al. Characterization of a first-pass gradient-echo spin-echo method to predict brain tumor grade and angiogenesis. AJNR Am J Neuroradiol 2004;25:1524-32 Medline

18. Mangla R, Kolar B, Zhu T, et al. Percentage signal recovery derived from MR dynamic susceptibility contrast imaging is useful to differentiate common enhancing malignant lesions of the brain. AJNR Am J Neuroradiol 2011;32:1004-10 CrossRef Medline

19. Quarles CC, Ward BD, Schmainda KM. Improving the reliability of 
obtaining tumor hemodynamic parameters in the presence of contrast agent extravasation. Magn Reson Med 2005;53:130716 Medline

20. Uematsu H, Maeda M, Sadato N, et al. Blood volume of gliomas determined by double-echo dynamic perfusion-weighted MR imaging: a preliminary study. AJNR Am J Neuroradiol 2001;22: 1915-19 Medline

21. Johnson G, Wetzel SG, Cha, et al. Measuring blood volume and vascular transfer constant from dynamic, $\mathrm{T}(2)^{*}$-weighted contrast-enhanced MRI. Magn Reson Med 2004;51:96-68 Medline

22. Heiland, Benner T, Debus J, et al. Simultaneous assessment of cerebral hemodynamics and contrast agent uptake in lesions with disrupted blood-brain-barrier. Magn Reson Imaging 1999;17:21-27 Medline

23. Welker K, Boxerman J, Kalnin A, et al; American Society of Functional Neuroradiology MR Perfusion Standards and Practice Subcommittee of the ASFNR Clinical Practice Committee. ASFNR recommendations for clinical performance of MR dynamic susceptibility contrast perfusion imaging of the brain. AJNR Am J Neuroradiol 2015;36:E41-51 CrossRef Medline

24. Boxerman J, Schmainda KM, Weisskoff RM. Relative cerebral blood volume maps corrected for contrast agent extravasation significantly correlate with glioma tumor grade, whereas uncorrected maps do not. AJNR Am J Neuroradiol 2006;27:859-67 Medline

25. Paulson ES, Schmainda KM. Comparison of dynamic susceptibilityweighted contrast-enhanced MR methods: recommendations for measuring relative cerebral blood volume in brain tumors. Radiology 2008;249:601-13 Medline

26. Semmineh NB, Stokes AM, Bell LC, et al. A population-based digital reference object (DRO) for optimizing dynamic susceptibility contrast (DSC)-MRI methods for clinical trials. Tomography 2017;3: 41-49 CrossRef Medline

27. Leu, K, Boxerman JL, Cloughesy TF, et al. Improved leakage correction for single-echo dynamic susceptibility contrast perfusion MRI estimates of relative cerebral blood volume in high-grade gliomas by accounting for bidirectional contrast agent exchange. AJNR Am J Neuroradiol 2016;37:1440 - 46 CrossRef Medline

28. Ellingson BM, Bendszus M, Boxerman J, et al; Jumpstarting Brain Tumor Drug Development Coalition Imaging Standardization Steering Committee. Consensus recommendations for a standardized brain tumor imaging protocol in clinical trials. Neuro Oncol 2015; 17:1188-98 CrossRef Medline

29. Hu LS, Baxter LC, Pinnaduwage DS, et al. Optimized preload leakage-correction methods to improve the diagnostic accuracy of dynamic susceptibility-weighted contrast-enhanced perfusion $\mathrm{MR}$ imaging in posttreatment gliomas. AJNR Am J Neuroradiol 2010;31: 40-48 CrossRef Medline

30. Kassner A, Annesley DJ, Zhu XP, et al. Abnormalities of the contrast re-circulation phase in cerebral tumors demonstrated using dynamic susceptibility contrast-enhanced imaging: a possible marker of vascular tortuosity. J Magn Reson Imaging 2000;11:103-13 Medline

31. Barajas RF Jr, Hodgson JG, Chang JS, et al. Glioblastoma multiforme regional genetic and cellular expression patterns: influence on anatomic and physiologic MR imaging. Radiology 2010;254:564-76 CrossRef Medline

32. Leu K, Enzmann DR, Woodworth DC, et al. Hypervascular tumor volume estimated by comparison to a large-scale cerebral blood volume radiographic atlas predicts survival in recurrent glioblastoma treated with bevacizumab. Cancer Imaging 2014;14:31 CrossRef Medline

33. Emblem KE, Bjornerud A, Mouridsen K, et al. T(1)- and $T(2)\left({ }^{*}\right)$ dominant extravasation correction in DSC-MRI: part II-predicting patient outcome after a single dose of cediranib in recurrent glioblastoma patients. J Cereb Blood Flow Metab 2011;31;2054-64 CrossRef Medline

34. Bjornerud A, Sorensen AG, Mouridsen K, et al. T1- and T2*-dominant extravasation correction in DSC-MRI, Part I: theoretical considerations and implications for assessment of tumor hemodynamic properties. J Cereb Blood Flow Metab 2011;31:2041-53 CrossRef Medline

35. Stokes AM, Semmineh N, Quarles CC. Validation of a T1 and T2* leakage correction method based on multiecho dynamic susceptibility contrast MRI using MION as a reference standard. Magn Reson Med 2016;76:613-25 CrossRef Medline

36. Schmiedeskamp H, Andre JB, Straka M, et al. Simultaneous perfusion and permeability measurements using combined spin- and gradient-echo MRI. J Cereb Blood Flow Metab 2013;33:732-43 CrossRef Medline 